\title{
Networking
}

\section{Contrasting approaches to recruitment in primary care research}

\author{
Elaine Ward ${ }^{1}$, Julia Miller ${ }^{2}$, Jonathan Graffy ${ }^{1,3}$ and Peter Bower ${ }^{2}$ \\ ${ }^{1}$ NIHR Primary Care Research Network, University of Leeds, UK \\ ${ }^{2}$ NIHR School for Primary Care Research, University of Manchester, UK \\ ${ }^{3}$ NIHR School for Primary Care Research, University of Cambridge, UK
}

\begin{abstract}
Aim: To describe approaches to recruitment, key challenges and strategies to improve recruitment among research organizations in the UK. Background: Recruiting research participants is challenging. Less than one-third of studies recruit to target on time. Methods: Descriptive survey with 31 participants from 22 public and private sector organizations. Findings: We identified a range of recruitment pathways, highlighting the extensive range of activities required throughout the process. Methods reported to improve recruitment were related to project management, context and resources. There were differences in emphasis between sectors concerning prioritization of staff roles, feasibility work and marketing. Conclusions: Organizations involved in primary care research adopt diverse approaches, yet cross-fertilization between groups is limited
\end{abstract}

Key words: organization and administration; primary care; research; research design; resources

Received 17 February 2009; accepted 24 July 2009; first published online 14 August 2009

\section{Introduction}

Difficulties in achieving recruitment targets on time are widespread (McDonald et al., 2006; Bower et al., 2007; Campbell et al., 2007a). Strategies applied to one study at a particular time may not work in another (Tognoni et al., 1991; Hunt et al., 2001). Only a one-third of studies attain recruitment targets (McDonald et al., 2006; Bower et al., 2007; Campbell et al., 2007a), with implications for the costs and validity of research.

There is little evidence in the public domain about successful strategies. Explanations for success or failure are largely anecdotal due to the

Correspondence to: Elaine Ward, 100a Ashley Gardens, Thirleby Road, London SW1P 1HJ, UK. Email: Elaine. forwardsolutions@gmail.com lack of rigorous tests of different strategies (Bryant and Powell, 2005; Watson and Torgerson, 2006; Rendell et al., 2007; Graffy et al., 2008). Yet an enormous amount of resource and effort goes into research in primary care in both public and private sectors. In 2006, the National Institute for Health Research (NIHR) Primary Care Research Network (PCRN) was established as part of the UK Department of Health's (2006) 'Best Research for Best Health' strategy. The objectives of PCRN, one of the NIHR Clinical Research Networks, are to facilitate the conduct of academic or industry-led clinical studies and timely participant recruitment in primary care.

However, a lack of reliable evidence about what enhances recruitment in specific circumstances remains, and researchers must rely on experience, rather than evidence. There have been calls to develop a more rigorous evidence 
base concerning the most effective methods of recruitment (Watson and Torgerson, 2006; Bower et al., 2009). However, the area of recruitment is also undergoing rapid change, with new organizations, allegiances, guidance and regulations. Evaluating recruitment methods requires an understanding of the processes underlying recruitment, and the context into which any new interventions will be introduced (Campbell et al., 2007b).

The aim of this study was to survey a range of informants involved in recruitment in primary care, to develop an understanding of the current context, and to explore current recruitment strategies, challenges and methods of overcoming them.

\section{Methods}

A sample of informants who were experienced in primary care research recruitment was recruited using snowballing techniques, personal contacts, conference workshops and the NIHR Clinical Research Networks.

Open-ended interviews were conducted by telephone or face to face. Participants were asked about their own recruitment experiences and the challenges they encounter. Discussions were recorded in note form and summarized to capture key elements and procedures. Participants were later e-mailed a summary of the approaches they reported and were asked to verify or elucidate this further.

\section{Findings}

Thirty-one participants contributed from 22 organizations, half of which were based in London and the remainder based in Greater Manchester, Oxfordshire, the East Midlands, Central England, Hertfordshire, Buckinghamshire, Lancashire, Cambridgeshire and Yorkshire. They included people working in academic primary care, commercial organizations conducting pharmaceutical research, NIHR research networks, general practice, and clinical trials units, some working in multiple settings. Informants included professors, clinicians, nurses, social scientists and research managers.

\section{Recruitment methods and the role of recruitment organizations}

Respondents distinguished two recruitment stages: collaborators (those acting as gatekeeper to participants ${ }^{1}$ ) and participants themselves. Most of them recruited with support from health service collaborators. Several reported examples of 'hub and spoke' models with a network of collaborators referring potential participants to a hub where the actual research takes place. Advertising direct to the public was more usual in industry. Recruitment methods were as dependent on the organization as much as on the nature of the study. Organizational factors affecting recruitment practices include: professional and cultural orientation (eg, driven by commercial, academic or network mores), resources, staffing structure and previous experiences of recruitment.

Industry organizations focus mainly on strategic planning and marketing. Informants from NIHR and MRC networks, clinical trial units and general practitioner (GP) practices describe their activities as facilitating research, using strategies involving employing staff such as research nurses. Despite sometimes being costly, several academics reported that using a research champion and putting resources into building relationships with collaborators and participants was particularly worthwhile. Industry also reported using this strategy, but do not necessarily stress its importance to the same degree. Informants highlight the worth of different recruitment procedures. For example, industry participants emphasized conducting tightly managed and monitored prestudy feasibility assessments. In contrast, academic or NIHR network informants prioritize activities such as identifying potential participants by searching health records.

Techniques used to identify suitable participants vary depending on the study. These include record searches using remote-access and near-patient electronic mechanisms, consultation-based approaches, recruitment through an intermediary or eliminating the need for NHS help by advertising direct to the public. For studies on 'hard-to-reach'

\footnotetext{
${ }^{1}$ Access to patient data is subject to the data protection act. Researchers may not directly access personal information about patients but usually rely on collaborators to identify patients for them and make requests for participation on their behalf.
} 


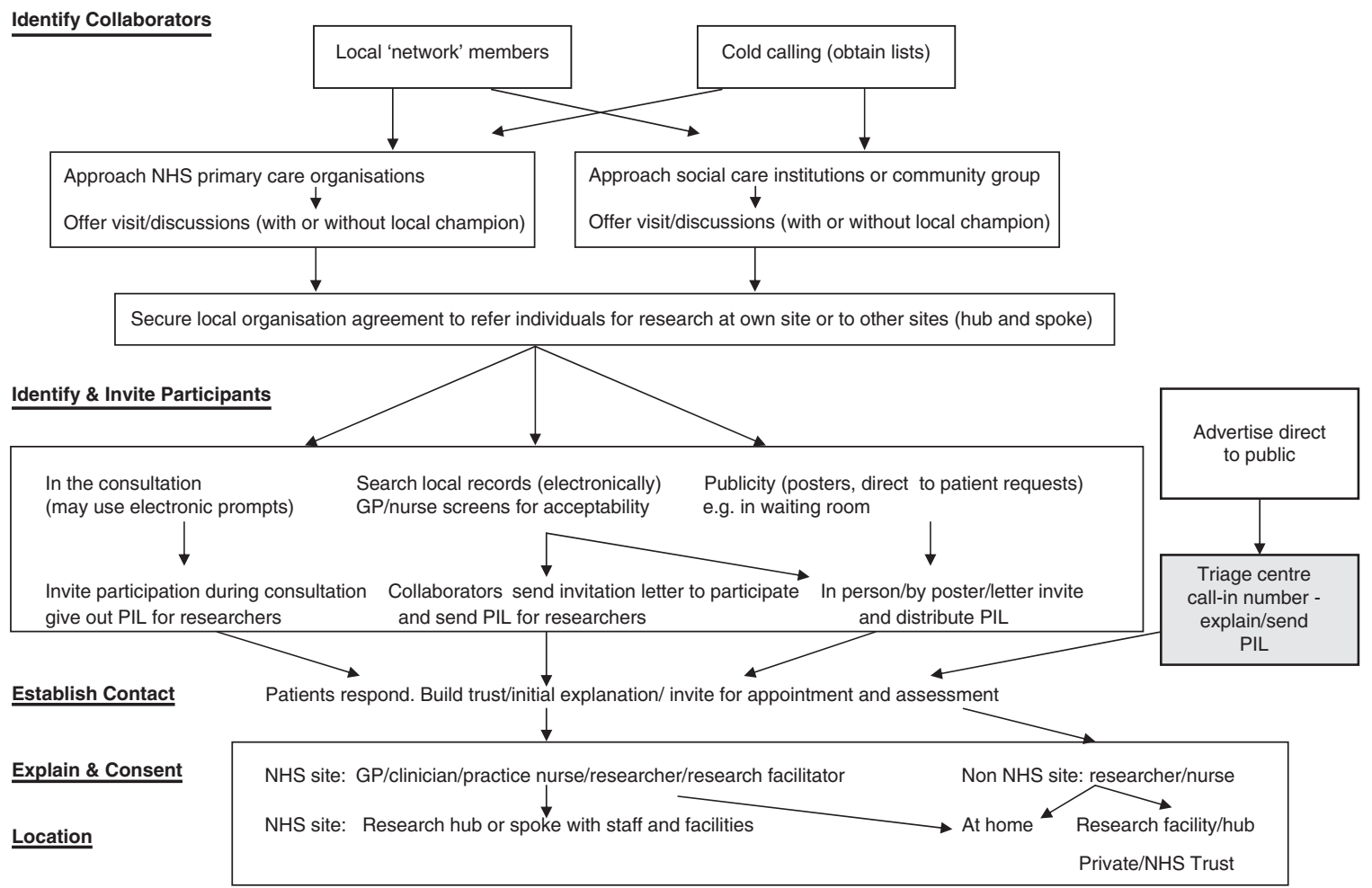

Key: PIL = patient information leaflet

Figure 1 Primary care research recruitment flowchart

populations such as minority groups or the vulnerable, help from intermediaries, such as spiritual leaders or care-home directors can be sought to promote the study to their communities.

Respondents stressed the need to tailor recruitment strategies to specific study requirements and the target groups' particular requirements. For example, suitable outreach locations such as mosques, libraries or other community settings may be needed, or particular needs can be met by large print, audio technologies, translations, Braille or other special data collection formats. Possible recruitment pathways are illustrated in Figure 1.

\section{Fitting research into clinical practice}

For research relying on clinicians to recruit, there is a need for well-informed, motivated clinical collaborators, with time to explain what a study involves during consultations. Some informants reported unease among clinicians when acting on behalf of the research team to recruit patients for research, because collaboration can introduce two difficulties. First, having to discuss treatment uncertainties in detail (the reason the research is being done in the first place) may conflict with the process of negotiating a care plan. Secondly, introducing the study to their patients means they may be questioned about methodological or other aspects of research, and they may be concerned that they lack sufficient knowledge. Informants reported that a lack of rewards and time pressures also deter involvement. Conversely, training, potential reductions in drugs or referral budgets, patient benefit or financial rewards were factors that were reported to motivate clinicians. Solutions to GP involvement include setting up dedicated research facilities or employing on-site dedicated research 
nurses, which facilitates time to explain studies, obtaining informed consent, intervention delivery and eliminates confidentiality problems.

\section{Marketing research}

Communication, marketing and media skills are stressed as important, particularly by those with industry connections. However, such processes are reportedly under-resourced outside industry. Personnel with good interpersonal skills and an understanding of both research and the primary care context are reportedly able to enhance recruitment by building and maintaining relationships with both collaborators and participants. Informants also suggest that studies need to have a strong identity reinforced by materials with a strong visual impact. Commercial organizations are able to put more resources into preparatory activities by conducting social marketing or using communications specialists to help them understand target groups and tailor study materials. This approach has recently been endorsed in the literature (Campbell et al., 2007a). Several informants emphasized the need for study selection on the basis of relevance and importance to both health professionals and patients. The value of a local champion to act as advocate for the study, was highlighted.

\section{Methods of improving recruitment}

As in the introduction, the actual scientific evidence concerning recruitment methods is sparse. However, through experience, respondents identified a number of recruitment methods that they

- Undertake pre-study market research - for insight into your audiences, prevalence and where are the best places to recruit (PPI input) $[\mathrm{P}, \mathrm{C}, \mathrm{R}]$

- Tailor communications to audience needs/agenda [C]

- Ensure study's relevance to local organisations/individuals [C]

- Identify issues which may impact co-operation (conflict and equipoise) $[P, C]$

- Identify how the study will 'fit' with healthcare delivery circumstances and structures [C]

- Plan processes which minimise collaborator workload $[P, C, R]$

- Organise research as separate from clinical care, if possible $[P, R]$

- Use network membership or previous links for intelligent targeting $[P, R]$

- Use local champions as ambassadors to stimulate collaboration [C,R]

- Provide support for collaborators (training, regular contact, advice) $[P, R]$

- Maximise participant identification by combined/selected methods: electronic searches, direct to community, in-situ during consultation, campaigns $[P, C, R]$

- Use screening for suitable participants and acquire consent for details to be kept on a database of willing participants for the future [R]

- Offer opportunities and rewards (health feedback, training, extra resources) $[P, R]$

- Build in participant-friendly strategies (flexible appointments, arrange travel) [P,C]

- Use expert, dedicated, trained research staff [P,R]

- Establish dedicated research units, manned by dedicated research staff $[P, R, C]$

- Reimburse costs of involvement and pay promptly [R,P]

- Plan for slow start-up, seasonality and other predictable delays [P]

- Include contingency plans for low recruitment $[P, R]$

- Develop/maintain good relationships $[\mathrm{P}, \mathrm{C}, \mathrm{R}]$

Figure 2 Recruitment tactics considered to be effective tactics are categorized by project planning (P), issues relating to context $(\mathrm{C})$ and ensuring appropriate resources are available (R)

Primary Health Care Research \& Development 2009; 10: 368-373 
reported as effective (Figure 2). These have been broadly categorized into three groups: project management including planning and preparation, addressing contextual factors and resources.

\section{Discussion}

\section{Summary}

This descriptive survey of recruitment organizations demonstrates the range of different approaches to recruitment in primary care, highlights the range of challenges and identifies some areas where there is some difference in focus between academic and non-academic organizations.

\section{Limitations of the study}

The strength of this pragmatic study is that it provides detailed descriptive information from expert informants engaged in day-to-day recruitment activities. However, as with any nonrandom recruitment method, there may be bias in the respondent sample. In addition, respondents were allowed to dwell on their preferred issues. The data were not recorded verbatim, fully transcribed or subject to a full qualitative analysis, and the subjective experience of respondents does not demonstrate effectiveness of any given approach.

\section{Implications for policy and practice}

There is considerable variability in approaches, tactics and key factors emphasized by informants. Social marketing (Kotler and Zaltman, 1971; Lefebvre and Flora, 1988; UK Department of Health, 2004) is more used by industry, while the use of networks, dedicated research staff and electronic searches seems more prevalent in academia. Industry stresses preparation and presentation, whereas academia emphasizes personnel and technical aids to facilitate recruitment. However, both sectors value good interpersonal skills and effective project management.

Without accurate data, it is difficult to determine which approaches are most effective although there is clearly potential for the different sectors to share learning and experiences, and to develop a model which takes account of different strategies employed, taking the best of each and factoring in an appraisal of the needs and resources of each individual project. The costs of marketing and quality communications advice may be high, but it may be possible for the NIHR to develop in-house training and expertise on which academics and other non-commercial researchers can call.

Even experienced and efficiently structured organizations find research recruitment in primary care challenging. Our data demonstrate the complexity of the research recruitment process and point to the importance of planning and practical steps. Strategies reported here are often more related to organizational processes and logistics rather than to science, suggesting that having the ability to present and 'sell' the research is as important as having research skills (as highlighted by Campbell et al. (2007a; 2007b)). Practical and operational processes - from how to identify, approach and consent potential participants and what or who to use for these - are additional issues. Each of these issues raises particular challenges for research into recruitment. For example, conventional trials could examine different ways of approaching patients, whereas comparisons of different approaches to marketing may fit less well into such a model.

Most of our informants report working through NHS primary care. It is generally assumed that research can be integrated into the clinical setting, and indeed, current regulatory arrangements are such that recruitment in clinical settings is often required. However, there were examples from both industry and academia where there was a move to separate research activities from the clinical consultation, and there are potential advantages of such an approach. However, these data do not allow us to determine if separation has a direct, positive impact on recruitment.

In summary, this paper presents a 'snapshot' of current recruitment process and methods in primary care, enumerates strategies in common use to enhance primary care recruitment, and will provide useful context for future research and the development and testing of recruitment interventions. Recruitment may profit from exchange of ideas and experiences between different organization and sectors. However, further work is needed to evaluate and test the contribution of specific recruitment strategies if the problems are to be overcome in a systematic and generalizable way. 


\section{Acknowledgements}

This work was supported by the National Institute of Health Research (School for Primary Care Research and Primary Care Research Network). The views expressed in this paper are those of the researchers and not the NIHR. The authors wish to acknowledge the advice and support of Brendan Delaney, Paul Wallace, Ann Louise Kinmonth, David Collier and Gill Sarre. This work took the form of informal dialogue with stakeholders; discussions were not taped and no direct quotes have been used, therefore ethics permission was not required.

\section{References}

UK Department of Health. 2004: Choosing health: making healthy choices easier. London: Stationery Office (CM 6374).

UK Department of Health (Research and Development Directorate). 2006: Best Research for Best Health: a new national health research strategy. London: HMSO.

Bower, P., Wallace, P., Ward, E., Graffy, J., Miller, J., Delaney, B. and Kinmonth, A.L. 2009: Improving recruitment to health research in primary care. Family Practice; doi: 10.1093/fampra/cmp03.

Bower, P., Wilson, S. and Mathers, N. 2007: How often do UK primary care trials face recruitment delays? Family Practice 24, 601-03.

Bryant, J. and Powell, J. 2005: Payment to healthcare professionals for patient recruitment to trials: systematic review. British Medical Journal 331, 1377-78.

Campbell, M.K., Snowdon, C., Francis, C., Elbourne, D., McDonald, A.M., Knight, R., Entwhistle, V., Garcia, J.,
Roberts, I. and Grant, A. 2007a: Recruitment to randomised trials: strategies for trial enrolment and participation study. The STEPS study. Health Technology Assessment 2007, 11.

Campbell, N., Murray, E., Darbyshire, J., Emery, J., Farmer, A., Griffiths, F., Guthrie, B., Lester, H., Wilson, P. and Kinmonth, A.L. 2007b: Designing and evaluating complex interventions to improve health care. British Medical Journal 334, 455-59.

Graffy, J., Grant, J., Boase, S., Ward, E., Wallace, P., Miller, J. and Kinmonth, A.L. 2008: UK research staff perspectives on improving recruitment and retention to primary care research; nominal group exercise. Family Practice 26, $48-55$.

Hunt, C.J., Shepherd, L.M. and Andrews, G. 2001: Do doctors know best? Comments on a failed trial. Medical Journal of Australia 174, 144-49.

Kotler, P. and Zaltman, G. 1971: Social marketing: an approach to planned social change. Journal of Marketing 35, 3-12.

Lefebvre, R.C. and Flora, J.A. 1988: Social marketing and public health intervention. Health Education Quarterly 15, 300-301.

McDonald, A., Knight, R. and Campbell, M. 2006: What influences recruitment to randomised controlled trials? A review of trials funded by two UK funding agencies. Trials 7, 9. doi: 10.1186/1745-6215-7-9.

Rendell, J, Merritt, R. and Geddes, J. 2007: Incentives and disincentives to participation by clinicians in RCTs. Cochrane Database of Systematic Reviews. doi: 10.1002/ 14561858.MR000021.pub3.

Tognoni, G., Alli, C., Avanzini, F., Bettelli, G., Colombo, F., Corso, R., Marchioli, R. and Zussino, A. 1991: Randomised clinical trials in general practice: lessons from a failure. British Medical Journal 303, 969-71.

Watson, J. and Torgerson, D. 2006: Increasing recruitment to RCTs: a review of RCTs. BMC Medical Research Methodology 6, 34. 